\title{
Synthesis of Gelsenicine
}

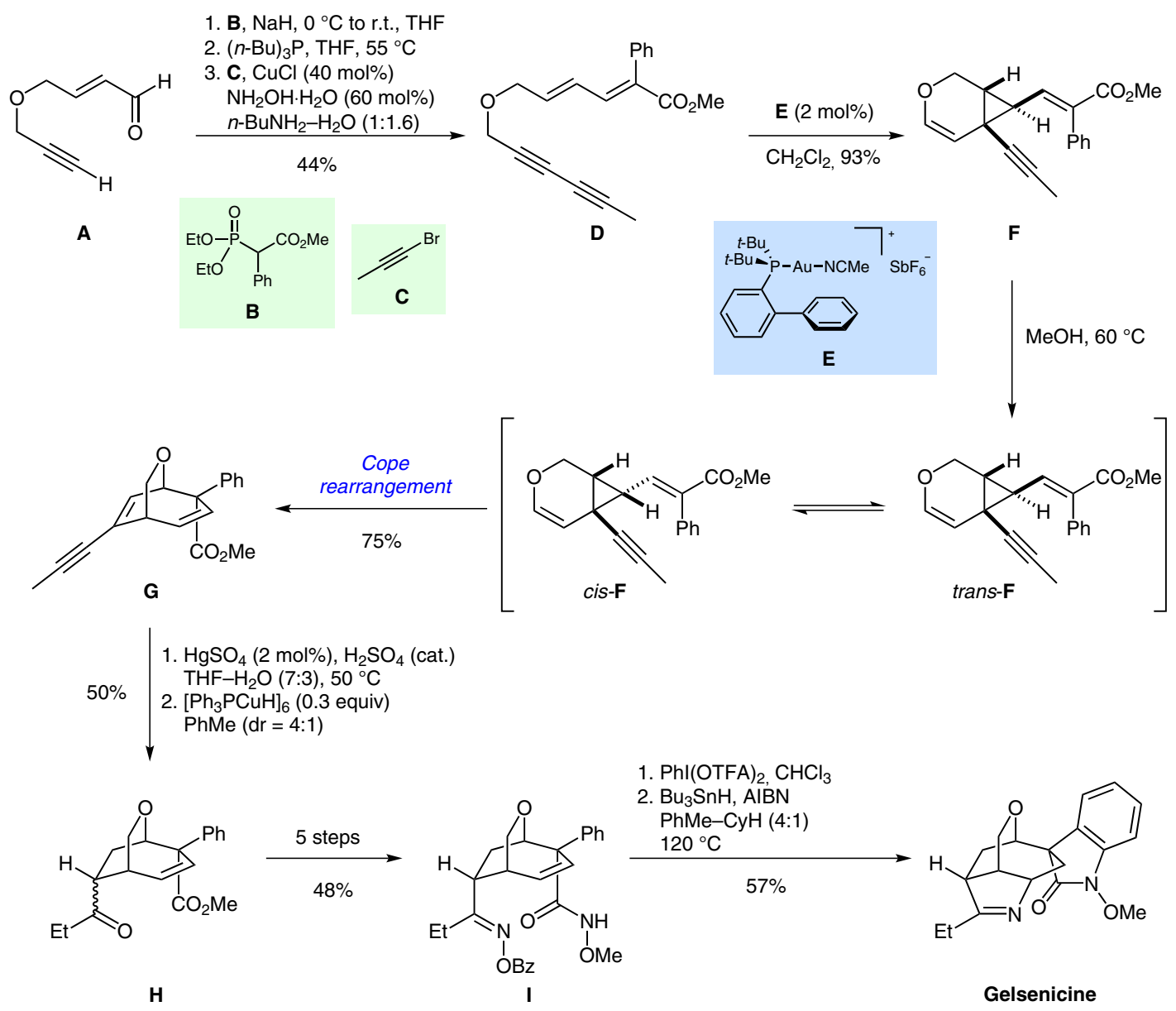

Significance: Gelsenicine is an oxindole alkaloid isolated from Gelsemium Elegans, a toxic plant widely distributed in Southeast Asia and used in traditional Chinese medicine. The structure features an oxabicyclo[3.3.2] system, an oxindole, and a pyrroline. Ferreira and co-workers disclose the first total synthesis of gelsenicine relying on a gold-mediated cycloisomerization followed by a Cope rearrangement that enabled the quick construction of the gelsenicine core.
Comment: Cycloisomerization precursor $\mathbf{D}$ was prepared from known aldehyde A using HornerWadsworth-Emmons olefination followed by Cadiot-Chodkiewicz coupling. Upon exposure to [Au(JohnPhos)(MeCN)SbF 6 ] (E), D underwent cycloisomerization to F. Heat-induced cis/trans isomerization and subsequent Cope rearrangement provided $\mathbf{G}$. $\mathrm{HgSO}_{4}$-mediated regioselective alkyne hydration followed by oxindole and radical pyrroline formation culminated in the synthesis of gelsenicine in 13 steps and $4.2 \%$ overall yield.

SYNFACTS Contributors: Erick M. Carreira, Marco Brandstätter 